\title{
Metabolic cooperation between co-cultured lung cancer cells and lung fibroblasts
}

\author{
Michael I Koukourakis ${ }^{1}$, Dimitra Kalamida', Achilleas G Mitrakas', Maria Liousia², Stamatia Pouliliou' ${ }^{1}$, Efthimios Sivridis ${ }^{2}$ \\ and Alexandra Giatromanolaki
}

Cooperation of cancer cells with stromal cells, such as cancer-associated fibroblasts (CAFs), has been revealed as a mechanism sustaining cancer cell survival and growth. In the current study, we focus on the metabolic interactions of MRC5 lung fibroblasts with lung cancer cells (A549 and H1299) using co-culture experiments and studying changes of the metabolic protein expression profile and of their growth and migration abilities. Using western blotting, confocal microscopy and RT-PCR, we observed that in co-cultures MRC5 respond by upregulating pyruvate dehydrogenase (PDH) and the monocarboxylate transporter MCT1. In contrast, cancer cells increase the expression of glucose transporters (GLUT1), LDH5, PDH kinase and the levels of phosphorylated/inactivated pPDH. H1299 cells growing in the same culture medium with fibroblasts exhibit a 'metastasis-like' phenomenon by forming nests within the fibroblast area. LDH5 and pPDH were drastically upregulated in these nests. The growth rate of both MRC5 and cancer cells increased in co-cultures. Suppression of LDHA or PDK1 in cancer cells abrogates the stimulatory signal from cancer cells to fibroblasts. Incubation of MRC5 fibroblasts with lactate resulted in an increase of LDHB and of PDH expression. Silencing of PDH gene in fibroblasts, or silencing of PDK1 or LDHA gene in tumor cells, impedes cancer cell's migration ability. Overall, a metabolic cooperation between lung cancer cells and fibroblasts has been confirmed in the context of direct Warburg effect, thus the fibroblasts reinforce aerobic metabolism to support the intensified anaerobic glycolytic pathways exploited by cancer cells.

Laboratory Investigation (2017) 97, 1321-1331; doi:10.1038/labinvest.2017.79; published online 28 August 2017

Aerobic usage of pyruvate is a key step for ATP production within the Krebs' cycle in normal cells. Cancer cells, however, avidly absorb glucose through upregulated transporters, ${ }^{1}$ that is subsequently transformed to pyruvate and lactate, bypassing the Krebs' cycle. This oxygen independent glycolysis described by Warburg several decades ago as 'aerobic glycolysis' characterizes the cancer cell metabolic behavior. ${ }^{2}$ The reason of this metabolic predilection remains poorly understood, although micro-environmental or oncogenic Akt and HIF gene upregulation may be part of the puzzle. ${ }^{3}$ Epstein et $a l^{4}$ recently showed that the rapid energetic demands of a rapidly growing and migrating cancer cell are less effectively served by the oxidative phosphorylation, as compared with the lactate pathway. Although the Krebs cycle produces a higher number of ATP molecules, the lactate pathway is faster and also produces high levels of glycolytic intermediates to support anabolic reactions in cells. ${ }^{5}$

The high amounts of lactate produced by aerobic glycolysis and the intracellular acidosis may become lethal for cancer cells, which is prevented by the upregulation of monocarboxylate transporters (MCTs) that extrude lactate outside the cancer cells. ${ }^{6}$ As an impaired intratumoral blood flow and the lack of a lymphatic network ${ }^{7}$ do not support lactate removal from the extracellular ambient, excessive extracellular acidosis may well lead to cell necrosis. In previous studies, we suggested that the tumor-associated stroma and cancerassociated fibroblasts (CAFs), well known to be an active tumor compartment that promotes growth and invasion, ${ }^{8}$ have an additional critical role by buffering the lactate produced by cancer cells. ${ }^{9-11}$ An interesting opposing hypothesis, the so called 'reverse Warburg effect', wants CAFs to be the glycolytic compartment in the tumor mass that provide aerobic cancer cells with lactate and metabolism products necessary for their growth. ${ }^{12}$

In the current study, we examine the paracrine interactions between lung fibroblasts and lung cancer cells growing within the same culture medium by studying effects on cancer cell growth and migration. We further investigate the metabolic

${ }^{1}$ Department of Radiotherapy/Oncology, Democritus University of Thrace, Alexandroupolis, Greece and ${ }^{2}$ Department of Pathology, Democritus University of Thrace, Alexandroupolis, Greece

Correspondence: Professor MI Koukourakis, MD, Department of Radiotherapy/Oncology, Democritus University of Thrace, PO Box 12, Alexandroupolis 68100, Greece. E-mail: targ@her.forthnet.gr

Received 8 May 2017; revised 17 June 2017; accepted 19 June 2017 
changes lung cancer cells and normal lung fibroblasts undergo when grown in co-cultures, trying to provide evidence on whether such a metabolic cooperation indeed occurs in the context of a direct or a reverse Warburg effect.

\section{MATERIALS AND METHODS \\ Cell Lines}

A549 (human alveolar basal epithelial adenocarcinoma cells), NCI-H1299 (human non-small cell lung carcinoma cells) and MRC5 (lung fibroblast cell line) were purchased from ATCC (ATCC CCL-185, ATCC CRL-5803 and ATCC CCL-171, respectively), and all experiments were performed within 6 months. Details on the cell lines can be found at https:// www.atcc.org/Products/All/CCL-185.aspx?slp = 1\#generalinforma tion and at http://www.atcc.org/Products/All/CRL-5803.aspx\#char acteristics and authentication of cell lines has been performed by ATCC and details and methods of characterization are available at http://www.atcc.org/Products/Cells_and_Microor ganisms/Testing_and_Characterization/STR_Profiling_Analysis. aspx. All three cell lines were cultured in DMEM culture medium $(+10 \%$ fetal bovine serum, 1\% L-Glutamin, Gibco) at constant conditions $37^{\circ} \mathrm{C}$ and $5 \% \mathrm{CO}_{2}$.

\section{Co-Culture Growth Assessment}

Equal number of lung cancer cells (A549 or H1299) and lung fibroblasts (MRC5 cells) were plated in two compartment tissue culture-treated petri dishes (KISKER, Germany). The cells were either plated as sibling co-cultures (A549/A549, H1299/H1299 and MRC5/MRC5) or as dissimilar co-cultures with shared culture medium (A549/MRC5 and H1299/ MRC5). Cells were incubated for $48 \mathrm{~h}$ at standard conditions, $37{ }^{\circ} \mathrm{C}$ and $5 \% \mathrm{CO}_{2}$ in humidified atmosphere. Following the incubation period, the numbers of cells were counted to assess the growth rate using a Hemocytometer and the Trypan Blue assay (Gibco, Thermo Fisher Scientific, USA) that identifies viable cells. The experiments were performed three times in order to confirm the significance of the results.

\section{D Co-Cultures}

Initially, the cell lines being used to generate spheroids were cultured in its normal growth culture medium (DMEM) as a two-dimensional (2D) monolayer. The cells were harvested at their exponential growth phase by trypsinization, and the cells were then counted using a hemocytometer. The cell numbers used for this experiment is 10000 cells per well; optimization for cell numbers per well was performed for spheroids' generation. Ultra-low attachment microplates and 96-well clear, round bottom sterile plates with lid (Corning no. 7007) have been used to grow individual spheroids. In the case of single culture spheroids, for both MRC5 and H1229 cell lines the spheroids have been created by spinning down 10000 cells at 1000 r.p.m. for $10 \mathrm{~min}$ and grown for 5 days. For cocultured spheroids, H1299 spheroids were initially generated and grown for 3 days and then they were further grown for
2 days in the presence of MRC5 cells, growing on transwell inserts, in the same culture medium. ${ }^{13}$

\section{Immunoblotting}

MRC5, A549 and H1299 cells were seeded in six-well culture plates. On top of the wells, special ThinCert Cell Culture Inserts (Greiner Bio-One 657640) were placed. These inserts have a capillary pore membrane $0.4 \mu \mathrm{m}$, TC surface treated. They allow the development of cell line co-cultures as two kinds of cell lines can be cultured on the bottom of the well separated by the insert and share the same medium. In this way, it is possible to examine the paracrine effects between cell lines. Co-cultures were seeded as sibling ((1) MRC5/ MRC5, (2) A549/A549 and (3) H1299/H1299) and as dissimilar co-cultures ((4) MRC5/A549 and (5) MRC5/ H1299). In this way, we could assess the protein levels in MRC5 cells (three conditions: when cultured as sibling, and when cultured with A549 or H1299 cells; symbolized as M, M $+\mathrm{A}$ and $\mathrm{M}+\mathrm{H}$ ), in $\mathrm{A} 549$ cells (two conditions: when cultured as sibling, or with MRC5 cells; symbolized as A and A+M) and in H1299 cells (two conditions: when cultured as sibling, or with MRC5 cells; symbolized as $\mathrm{H}$ and $\mathrm{H}+\mathrm{M}$ ). Protein cell lysates from each condition were collected after 48 and $96 \mathrm{~h}$ of co-cultured incubation in a sucrose buffer $(0.25 \mathrm{M}$ sucrose, $0.025 \mathrm{M}$ Tris- $\mathrm{HCl}, \mathrm{pH}$ 7.4) containing protease inhibitors (Roche Diagnostics $\mathrm{GmbH}$ ) and phosphatase inhibitors (Cell Signaling Technology). In all, $20 \mu \mathrm{g}$ of the whole protein lysates were separated on discontinuous SDS gels of $10 \%$ separation and 5\% stacking gels for all the proteins and transferred on PVDF membranes. Following blocking with $5 \%$ nonfat dry milk (for pPDH, the blocking stage in 5\% BSA-TBS-Tween $0.05 \%$ ), membranes were hybridized overnight with the following primary antibodies: Anti-Pyruvate Dehydrogenase E1-alpha subunit (phospho S293) antibody (1:1000, ab92696, Abcam, UK), Anti-Monocarboxylic acid transporter 1 antibody (1:500, ab85021, Abcam, UK) AntiGlucose Transporter GLUT1 antibody (1:500, ab15309, Abcam, UK), Anti-Pyruvate Dehydrogenase E1-alpha subunit antibody (9H9AF5) (1:1000, ab110330, Abcam, UK), Antipan PDK antibody (3H5BE12) (1:1000, ab115321, Abcam, UK), anti-Hexokinase II antibody (3D3) (1:500, ab104836, Abcam, UK), Anti-Lactate Dehydrogenase I antibody (1:1000, ab9001, Abcam, UK), and Anti-Lactate Dehydrogenase antibody V (1:1000, ab9002, Abcam, UK). The membranes were then hybridized with the appropriate secondary antibodies and developed in Amersham ECL Western blotting detection reagents and analysis system (RPN2209, GE Healthcare). Blot images were captured using the Chemidoc MP imaging system (Bio-Rad). All the blots were then stripped and rehybridized with a polyclonal antibody to b-Actin (1:5000; Nb 600-501; Novus Biologicals).

\section{RNA Extraction, cDNA Synthesis and qPCR}

Nucleospin RNA, (740955, Macherey-Nagel, Germany) was used for RNA extraction. A total of $10^{6}$ cells were collected 
after trypsinization and centrifugation at 1000 r.p.m. for $5 \mathrm{~min}$. For RNA harvesting, cells were resuspended in $350 \mu \mathrm{l}$ RA1 buffer and $3.5 \mu \mathrm{l} \beta$-mercaptoethanol. Then the steps of this kit were followed in order to achieve a high yield of RNA. The sample concentrations were measured using NanoDrop 2000c (Thermo Scientific, USA) and RNA samples were stored at $-80^{\circ} \mathrm{C}$.

In all, $1 \mu \mathrm{g}$ of RNA and Primerscript RT reagent kit (RR037A, Takara, France) was used for cDNA synthesis. First, a reaction that contains $500 \mathrm{ng}$ of RNA, $2 \mu \mathrm{l}(1 \times)$ of $5 \times$ PrimerScript Buffer, $0.5 \mu \mathrm{l}$ of PrimerScript Enzyme Mix I, $0.5 \mu \mathrm{l}(25 \mathrm{pmol})$ of Oligo dTs, $0.5 \mu \mathrm{l}(50 \mathrm{pmol})$ of random hexamers and $\mathrm{H}_{2} \mathrm{O}$ up to $10 \mu \mathrm{l}$ was prepared on ice. Afterwards, the reaction was incubated using the following conditions: $35^{\circ} \mathrm{C}$ for $15 \mathrm{~min}$ and $85^{\circ} \mathrm{C}$ for $5 \mathrm{~s}$. The cDNA samples were stored at $-20^{\circ} \mathrm{C}$.

In this study, the expression levels of the genes were measured using real-time quantitative PCR and the SensiMix SYBR No-ROX Kit (KAPA SYBR FAST qPCR Master Mix (2X) optimized for LightCycler ${ }^{\circledR} 480$ (KK4611, KapaBiosystems, USA)) was used. The reaction of $20 \mu \mathrm{l}$ was prepared adding KAPA SYBR FAST qPCR Master Mix $(2 \times)$ optimized for LightCycler ${ }^{\circledR} 480,200 \mathrm{nM}$ of forward primer and $200 \mathrm{nM}$ of reverse primer. The suggested cycle conditions were used: 1 cycle at $95^{\circ} \mathrm{C}$ for $15 \mathrm{~s}$ and 40 cycles at $95^{\circ} \mathrm{C}$ for $15 \mathrm{~s}, 56^{\circ} \mathrm{C}$ for $15 \mathrm{~s}$ and $72^{\circ} \mathrm{C}$ at 1 sec. All reaction conditions were performed on Roche Light Cycler 480II in triplicates. The primer sets that were used were designed using Roche Primer design tool and are presented in Table 1.

The relative changes in transcriptional levels of all genes between control and different conditions were calculated

Table 1 Primers used in this study

\begin{tabular}{|c|c|c|}
\hline Primer name & $\begin{array}{l}\text { Primer sequence }\left(5^{\prime} \rightarrow 3^{\prime}\right) \\
\text { Forward/reverse }\end{array}$ & Product size \\
\hline \multirow[t]{2}{*}{ LDHA } & GCAGATTTGGCAGAGAGTATAATG & $103 \mathrm{bp}$ \\
\hline & GACATCATCCTTTATTCCGTAAAGA & \\
\hline \multirow[t]{2}{*}{ LDHB } & GATGGATTITGGGGGAACAT & $104 \mathrm{bp}$ \\
\hline & AACACCTGCCACATTCACAC & \\
\hline \multirow[t]{2}{*}{ PDHA } & GTGCTGTCTGGCGCTTCT & $83 \mathrm{bp}$ \\
\hline & GACTGCTTGAGCCTGGAGAT & \\
\hline \multirow[t]{2}{*}{ PDK1 } & CACCAAGACCTCGTGTTGAG & $74 \mathrm{bp}$ \\
\hline & ACGTGATATGGGCAATCCAT & \\
\hline \multirow[t]{2}{*}{ MCT1 } & GTGACCATTGTGGAATGCTG & $66 \mathrm{bp}$ \\
\hline & CATGTCATTGAGCCGACCTA & \\
\hline \multirow[t]{2}{*}{ GLUT1 } & GGTTGTGCCATACTCATGACC & $66 \mathrm{bp}$ \\
\hline & CAGATAGGACATCCAGGGTAGC & \\
\hline \multirow[t]{2}{*}{ b-Actin } & CTAAGGCCAACCGTGAAAAG & $104 \mathrm{bp}$ \\
\hline & ACCAGAGCATACAGGGACA & \\
\hline
\end{tabular}

using the comparative $\mathrm{Ct}$ method $\left(2^{-\mathrm{ddCt}}\right)$ as the efficiencies of the target genes and reference gene are approximately equal near to $100 \%$. ActB was used as a reference gene for the normalization of the results. The equations that were utilized are presented below:

$\mathrm{RQ}=2^{-\mathrm{ddCt}}$,

$$
\begin{aligned}
\mathrm{ddCt}= & {\left[\left(\mathrm{Ct}_{\text {target }} \text { gene }-\mathrm{Ct}_{\text {referencegene }}\right)_{\text {sample }}-\right.} \\
& \left.\left(\mathrm{Ct}_{\text {target }} \text { gene }-\mathrm{Ct}_{\text {referencegene }}\right)_{\text {calibrator }}\right]
\end{aligned}
$$

\section{Silencing of Genes}

Human lung cancer cell lines A549 and H1299 and human normal fibroblast MRC5 were cultured and maintained using standard procedures. siRNAs were custom synthesized (GenePharma Co, Shanghai, China), pooled and used at $100 \mathrm{nM}$ to transfect cancer cells with PDK and LDHA siRNA and normal fibroblasts with DLAT(PDH) siRNA using Metafectene Pro (Biontex) for $24 \mathrm{~h}$; the silencing efficiency of siRNAs was confirmed both by immmunofluorescence and western blotting analysis after $48 \mathrm{~h}$. The siRNA used were as follows:

PDH siRNAs (siDLAT) were custom synthesized from Shanghai GenePharma Co., Ltd (China) and were pooled as (5'-GCCUGGAGGAGUGUUAUAUTT-3'), (5'-GGUACGGA AAGAACUUAAUTT- $\left.3^{\prime}\right)$, (5'-GGUCCCUGCAGAUAAUGAA TT- $3^{\prime}$ ) and (5'-GUGGCUUGCUGAGUUUAGAAT- $3^{\prime}$ ).

PDK1 siRNAs (siPDK1) were custom synthesized from Shanghai GenePharma Co., Ltd (China) and were pooled as (5'-GCCAAUACAAGUGGUUUAUTT-3'), (5'-GGAUGCUA AAGCUAUUUAUTT-3'), (5'-CCCAGGGUGUGAUUGAAU ATT- $\left.3^{\prime}\right)$ and ( $5^{\prime}$-CUGUCAGACUGGCAAAUAUTT- $\left.3^{\prime}\right)$.

LDHA siRNAs (siLDHA) were custom synthesized from Shanghai GenePharma Co., Ltd (China) and were pooled as (5'-CUGGCAAAGACUAUAAUGUTT- $\left.3^{\prime}\right), \quad$ (5'-GCCAUCAG UAUCUUAAUGATT-3'), (5'-CCAGUUUCCACCAUGAUU ATT-3') and (5'-GUGCUUAUGAGGUGAUCAATT-3').

\section{Confocal Microscopy}

For immunofluorescence staining, cells (A549, H1299 and MRC5) were grown on No. 1.5 glass coverslips, initially placed in petri dishes, transferred in appropriate combinations in single cultures and co-cultures for $48 \mathrm{~h}$, fixed in $3.7 \%$ paraformaldehyde/PBS $\mathrm{pH} 7.4$ for $20 \mathrm{~min}$ at $37^{\circ} \mathrm{C}$ and then permeabilized in PBS/0.1\% v/v Triton X-100 pH 7.4 for 5 min at room temperature. In addition, cells were blocked in PBS/ $5 \% \mathrm{w} / \mathrm{v}$ BSA pH 7.4 for $20 \mathrm{~min}$ and stained with the previously reported primary antibodies: anti-PDH E2/E3bp mouse monoclonal (1:1000, ab110333, Abcam, UK) antiPDH E1 phospho S293 rabbit polyclonal (1:500, ab92696, Abcam, UK), anti-Pan-PDK mouse monoclonal (1:200, ab115321, Abcam, UK), anti-LDH5 sheep polyclonal (1:200, 
ab9002, Abcam, UK), anti-LDH1 sheep monoclonal (1:200, ab9001, Abcam, UK), anti-GLUT1 rabbit polyclonal (1:100, ab15309, Abcam, UK), and anti-Hexokinase II mouse monoclonal (1:500, ab104836, Abcam, UK) for $1 \mathrm{~h}$ at RT. Cells were washed in PBS pH 7.4, incubated with appropriate CF 488 and 564 secondary antibodies at RT. DNA was counterstained with Hoechst $33342(1 \mu \mathrm{g} / \mathrm{ml}$; Sigma-Aldrich). After final washes, coverslips were mounted in homemade Mowiol mounting medium. Imaging was performed on a customized Andor Revolution Spinning Disk Confocal System built around a stand (IX81; Olympus) with a $60 \times$ lens and a digital camera (Andor Ixon+885) (CIBIT Facility, MBG-DUTH). Image acquisition was performed with the Andor IQ 2 software. Optical sections were recorded every $0.3 \mu \mathrm{m}$. All confocal microscopic images presented in this work are 2D maximum intensity projections of $z$-stack images (ImageJ $1.47 \mathrm{v}$ National Institute of Health, USA).

\section{Ki67 Confocal Microscopy}

Ki67 was assessed in 8 cell line conditions cultured as sibling cultures of wild type and shLDHA and shPDK1 cancer cells or shPDH MRC5 cells and in 12 cell line conditions of dissimilar co-cultures (Supplementary Table S1). Assessment was performed $48 \mathrm{~h}$ after cell co-culturing. Using the abovementioned technique, cells were stained with anti-Ki67 mouse monoclonal primary antibody (1:150; DAKO) for $1 \mathrm{~h}$ at room temperature. Image analysis and quantification of Ki67 expression in the nucleus have been performed using custom-developed macros in the Image software $1.47 \mathrm{v}$ (National Institute of Health, USA). At least five representative images obtained with confocal microscopy at $\times 100$ magnification were analyzed, so that expression of Ki67 was assessed in a cell number ranging from 20 to 56 (median 28). The $2 \mathrm{D}$ average projection of $z$-stack images were quantified using the nuclear area where integrated intensity values have been measured. Statistical analysis and graph presentation has been performed using the GraphPad Prism Version 5.01a statistical package (GraphPad Software Inc., USA).

\section{Migration Assay}

To assess the effect of silencing of key metabolism genes, namely, PDH for fibroblasts and LDHA and PDK1 for cancer cells, in the migration ability of cells in fibroblast/cancer cell co-cultures, the IBIDI Single Culture-Insert (IBIDI GmbH) in a $35 \mathrm{~mm} \mu$-Dish was used. This migration monitoring system consists of two reservoirs separated by a $500 \mu \mathrm{m}$-thick wall. Equal numbers of pairs of cells (control, DLAT(PDH)silenced, PDK-silenced and LDHA(V)-silenced) $7 \times 10^{5}$ cells/ $\mathrm{ml}$ were added into the two reservoirs of the same insert and incubated at $37^{\circ} \mathrm{C} / 5 \% \mathrm{CO}_{2}$. The pairs of cell lines used are shown in Supplementary Table S1.

After $24 \mathrm{~h}$, the insert was removed with caution creating a gap of $500 \mu \mathrm{m}$. The $35 \mathrm{~mm} \mu$-Dish has been finally filled with complete DMEM growth medium and the migration was monitored by bright-field microscopy at specific time points.
The images were analyzed using the TScratch software, developed by the Koumoutsakos group (CSE Lab), at ETH Zurich, the percentage of cell-free area was calculated and plotted using the GraphPad Prism 5.01 statistical package (GraphPad Software Inc., USA).

To abolish proliferation as a confusing factor in our migration experiments, cells were treated with Mitomycin C (KYOWA KIRIN) at $5 \mu \mathrm{g} / \mathrm{ml}$ for $3 \mathrm{~h}$, before the insert was removed.

\section{Graph Presentation and Statistics}

Graph presentation and statistical analysis was performed using the GraphPad Prism 5.01 version. Paired or unpaired two-tailed $t$-test was applied for analysis, as appropriate. A $P$-value of $<0.05$ was considered for significance.

\section{RESULTS}

\section{Metabolic Changes (Western Blotting)}

Following $48 \mathrm{~h}$ co-culturing of MRC5 fibroblasts with A549 or H1299 lung cancer cell lines, MRC5 cells responded by upregulating PDH (1.26- and 1.20-fold increase, respectively, $P<0.05)$ and MCT1 (1.41- and 1.40-fold increase, respectively, $P<0.01)$ expression, as shown in western blotting analysis (Figure 1a). LDH1 was strongly expressed in MRC5 fibroblasts and showed no changes when they were cocultured with cancer cells. LDH5 expression was less intense than LDH1 and remained stable in co-cultures. A slight, not significant, increase of pPDH was noted.

Both cancer cell lines responded in co-cultures by strongly upregulating proteins responsible for glucose absorption (GLUT1; 3.4- and 4.2-fold increase, respectively, $P<0.001$ ), anaerobic pyruvate usage, thus LDH5 (3.4- and 2.84-fold increase, respectively, $P<0.001)$ and PDK (1.24- and 1.34fold increase, respectively, $P<0.05)$, as well as lactate extrusion (MCT1; 1.8- and 2.4-fold increase, $P<0.05$ ). LDH1 expression was reduced (0.49- and 0.40-fold, respectively, $P<0.001)$ and PDH was also reduced in the A549 cell line (0.7-fold, $P<0.05)$. Phosphorylated (de-activated) pPDH was induced in both cancer cell lines (3.65- and 2.04-fold increase, respectively, $P<0.001)$.

\section{Metabolic Changes (Confocal Microscopy)}

Confocal microscopy confirmed the western blotting findings. LDH5 and PDK1 were strongly overexpressed in cancer cell lines when co-cultured with MRC5 fibroblasts (Figures $1 \mathrm{~b}$ and c). On the contrary, MRC5 fibroblasts sustained stable levels of the enzymes, while they increased their PDH levels when co-cultured with cancer cells on a background of low expression levels of pPDH (Figures 1d and e). MCT1 and GLUT1 was upregulated in co-cultured cancer cells. In fibroblasts, MCT1 was upregulated but GLUT1 levels did not change (Figures 1f and g). 
a

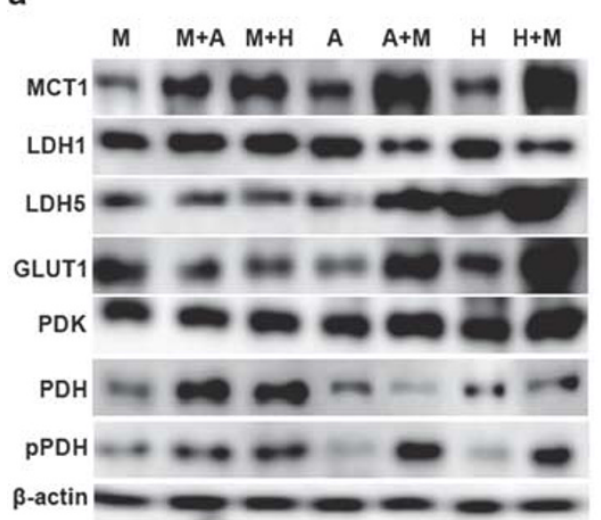

b

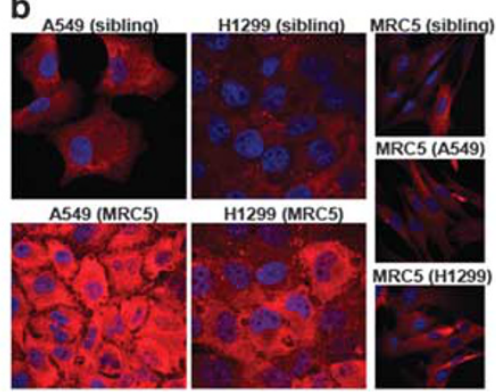

LDH5

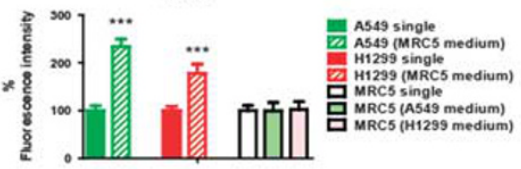

C

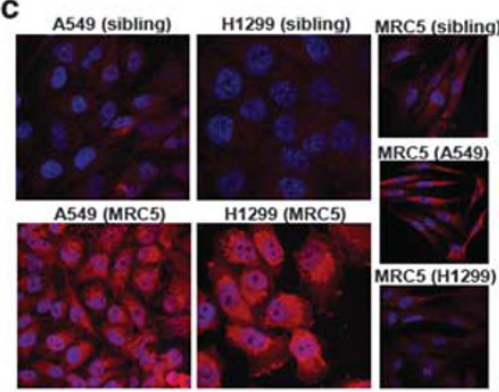

PDK1

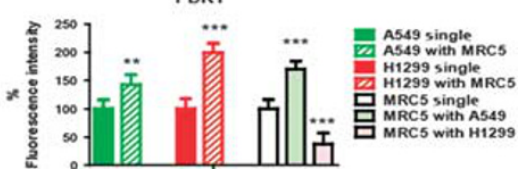

d

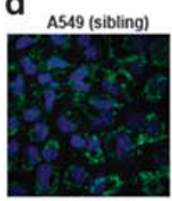

A549 (MRC5)
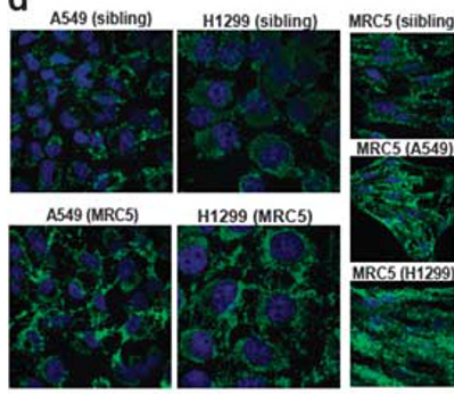

PDH

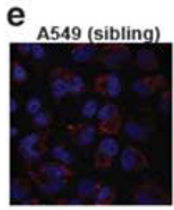

A549 (MRC5)

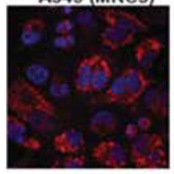

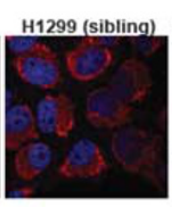

H1299 (MRC5)

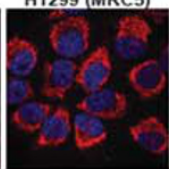

f
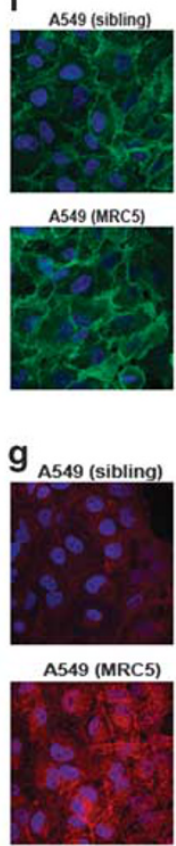

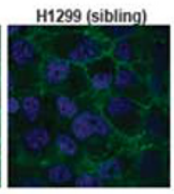

H1299 (MRC5)
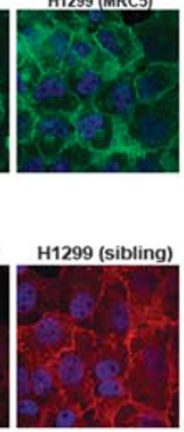

H1299 (MRC5)

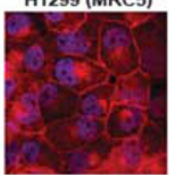

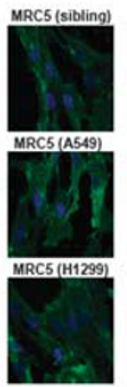

GLUT1

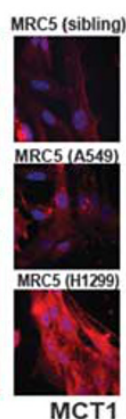

h
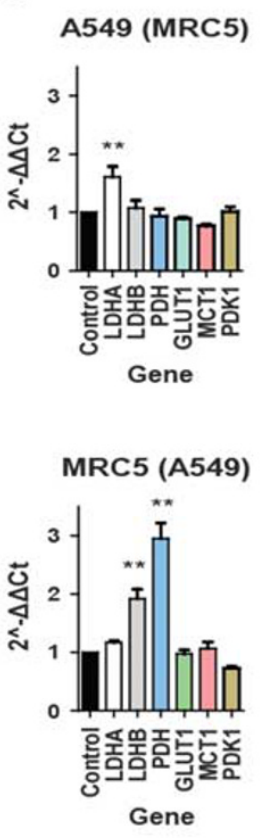

H1299 (MRC5)

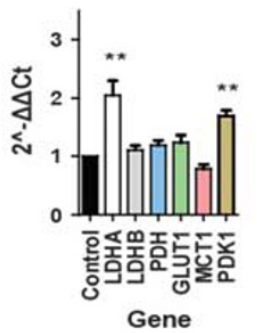

MRC5 (H1299)

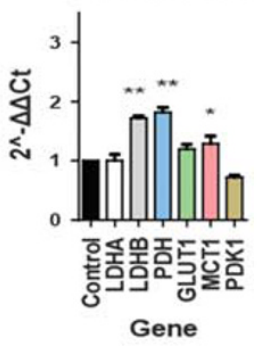

Figure 1 (a) Western blotting analysis of the expression of metabolism-related proteins in MRC5 (M) fibroblasts, A549 (A) and H1299 (H) lung cancer cells when cultured as single or adjacent cell populations (with common culture medium). The bands shown correspond to $45 \mathrm{kDa}$ for MCT1, $37 \mathrm{kDa}$ for LDH1 (LDHB) and LDH5 (LDHA), 52 kDa for GLUT1, 65 kDa for PDK1, 45 kDa for PDH E1 subunit and phosphorylated PDH. (b, c) Confocal microscopic images and fluorescence intensity analysis of the expression of LDH5 (b) and of PDK1 (c) in MRC5 fibroblasts, A549 and H1299 lung cancer cells when cultured as single or adjacent cell populations (with common culture medium). (d-g) Confocal microscopic images of the expression of PDH (d), pPDH (e), GLUT1 (f) and MCT1 (g) in MRC5 fibroblasts, A549 and H1299 lung cancer cells when cultured as single or adjacent cell populations (with common culture medium). (h) Quantitative RT-PCR detection of gene mRNA expression in MRC5 fibroblasts, A549 and H1299 lung cancer cells when cultured as single or adjacent cell populations (with common culture medium). ${ }^{*} P<0.05 ;{ }^{* *} P<0.01 ;{ }^{* *} P<0.001$.

\section{Metabolic Changes (RT-PCR)}

Quantitative RT-PCR supported the above observations at the protein level (Figure 1h). MRC5 fibroblasts responded in cocultures with cancer cells by significantly upregulating the mRNA expression of LDHB and the PDH genes. A significant overexpression of LDHA mRNA was noted in both A549 and H1299 lung cancer cell lines when growing in the same culture medium with MRC5 fibroblasts. PDK1 was also found to significantly increase in the H1299 cell line.

\section{Intra-Fibroblast Growing H1299 Nests}

Of interest, H1299 lung cancer cells growing in the same culture medium with fibroblasts exhibited a 'metastasis-like' phenomenon, the cancer cells growing as a nest within the fibroblast area of the co-culture. This was noted in repeated experiments for the $\mathrm{H} 1299$ cell lines but was never observed in the A549/MRC5 co-cultures. We therefore had the opportunity to examine the expression of LDH5 and $\mathrm{PDH} /$ $\mathrm{pPDH}$ in this 'mixed-co-culture' growing cell system. Using 
a
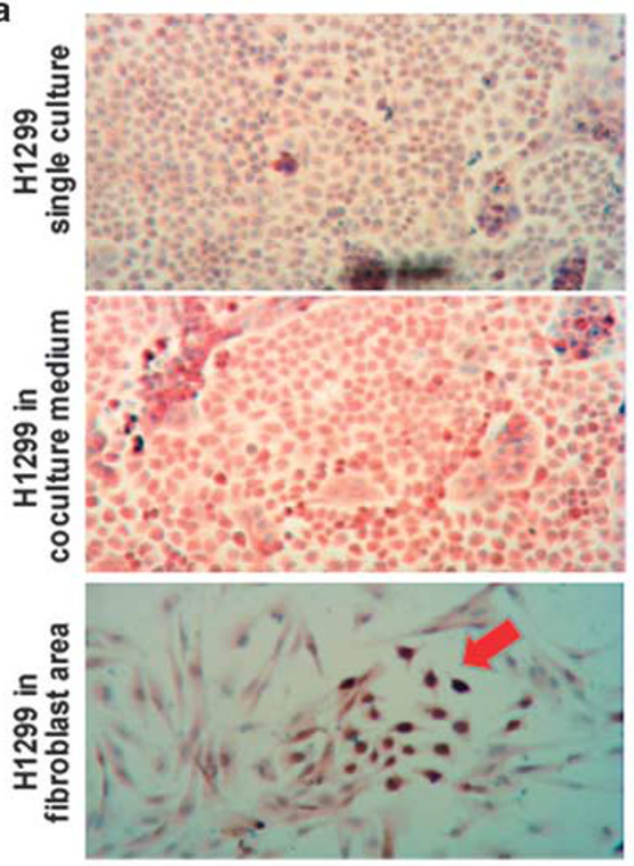

LDH5

b

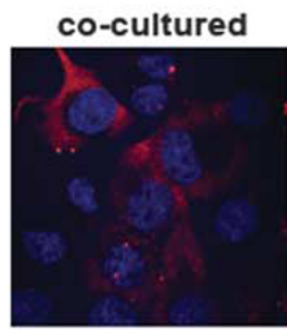

LDH5

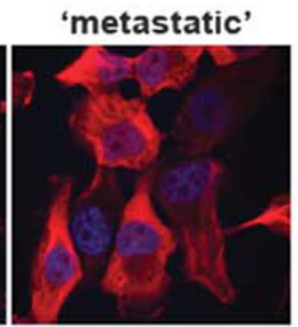

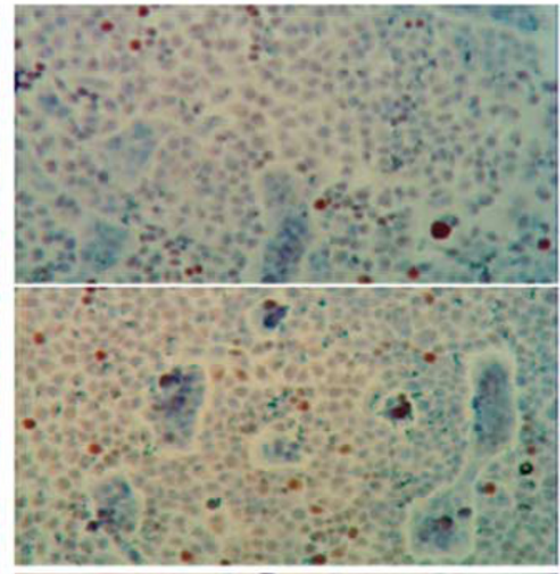

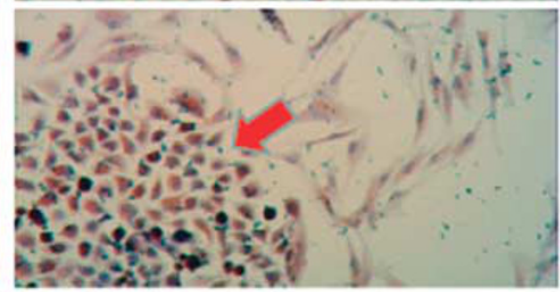

pPDH c

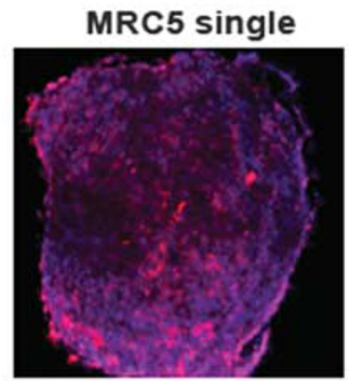

H1299 single

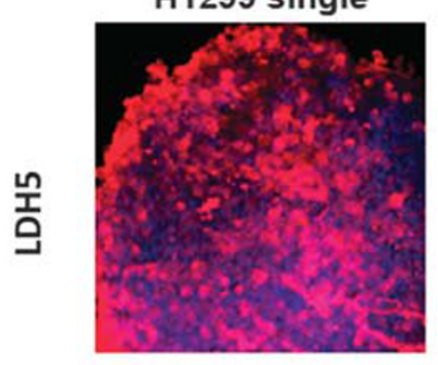

H1299 in MRC5
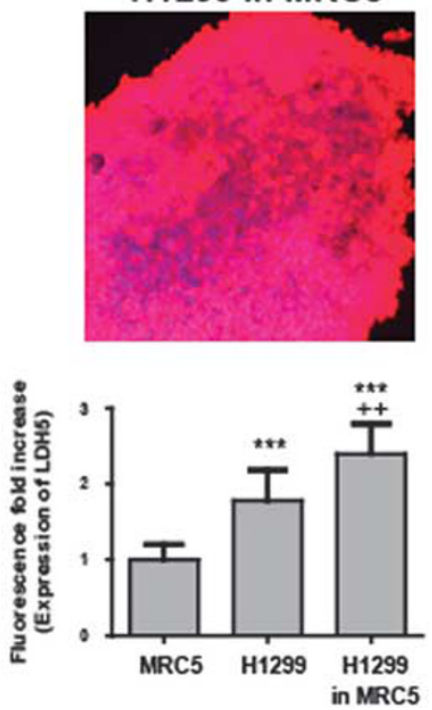

Figure 2 (a, b) Immunocytochemical (a) and confocal immunofluorescent (b) detection of LDH5 and of pPDH in H1299 cancer cells growing as single cultures, adjacent co-cultures with MRC5 fibroblasts sharing the same medium and as 'metastasis-like' cancer cell nests (red arrows) within the area of MRC5 fibroblasts. (c) Confocal image spheroids growing in 3D cultures immunostained for LDH5. Note the poor LDH5 expression in MRC5 and strong in H1299 spheroids. H1299 spheroids growing in MRC5 culture medium showed an intensification of LDH5 expression. Fluorescence intensity analysis of LDH5 expression is also shown ( ${ }^{* * *} P<0.001$ compared with MRC5; ++ compared with H1299 growing in standard medium).

immunocytochemistry, LDH5 and pPDH were upregulated in the H1299 cell lines and were further overexpressed in H1299 cell foci within the intra-fibroblast environment. No changes of their expression in the adjacent MRC5 fibroblasts was noted (Figures $2 \mathrm{a}$ and $\mathrm{b}$ ).

MRC5 and H1299 cancer cell line 3D cultures were established in standard culture medium. Moreover, H1299 spheroids growing in culture medium shared with MRC5 growing cells were developed. Immunofluorescence for $\mathrm{LDH} 5$ showed that MRC5 spheroids had a weak LDH5 expression, while H1299 spheroids showed an intense LDH5 reactivity $\left({ }^{* *} \mathrm{P}<0.001\right.$ compared with MRC5). H1299 Spheroids growing in MRC5 medium showed a further induction of LDH5 $(++P<0.05$ compared with $\mathrm{H} 1299$ cultured in standard culture medium) (Figure 2c).

\section{MRC5 Fibroblast Proliferation in Co-Cultures}

A direct comparison of the growth between sibling and dissimilar co-cultures growing in the same culture medium, as assessed with the Trypan Blue assay, showed intensified growth when cancer cells were cultured aside to lung fibroblasts for 2 days. This concerned both the fibroblasts and the cancer cells (Figure 3a). In accordance with the increased growth noted above, MRC5 fibroblasts and both 

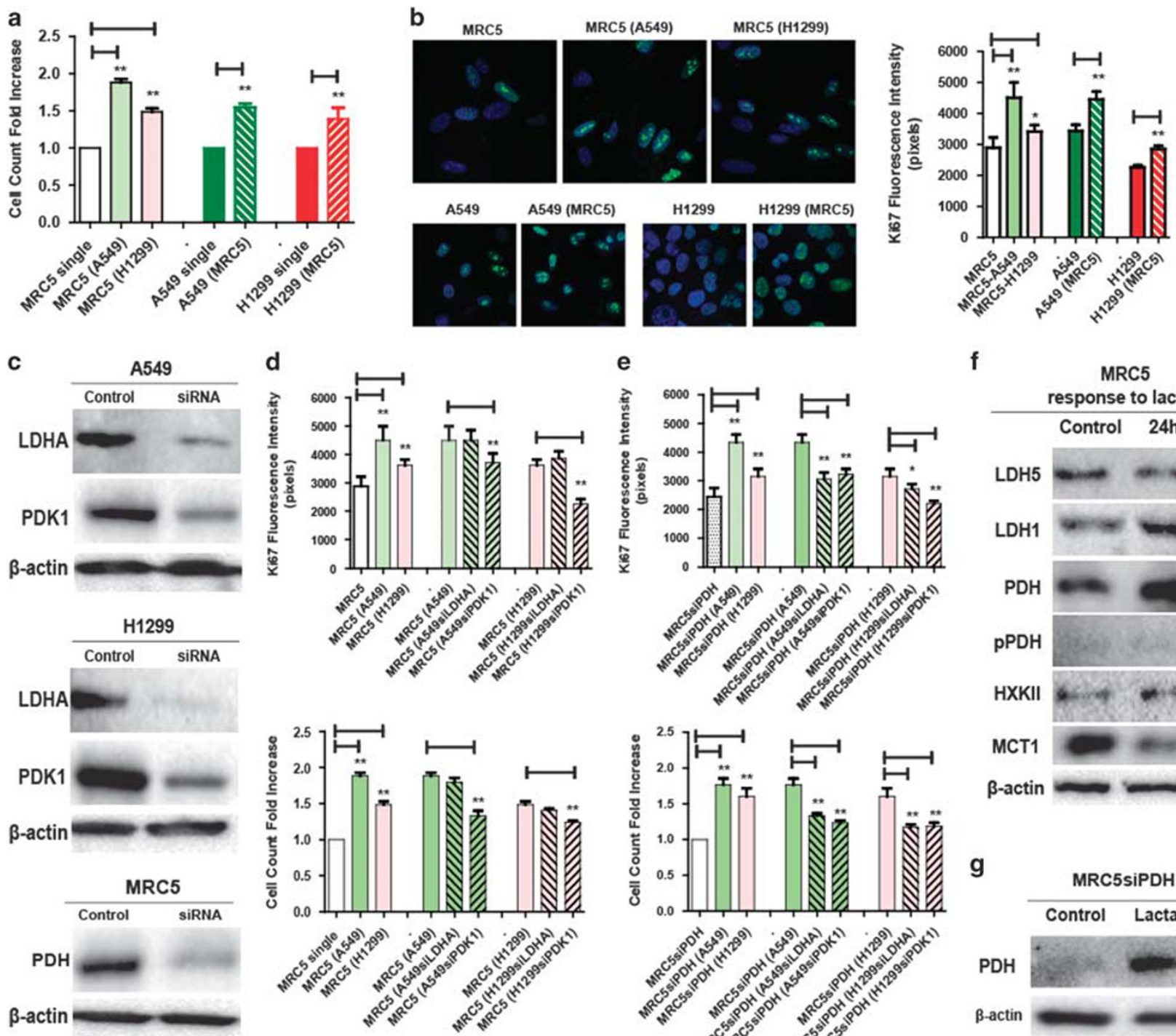

f
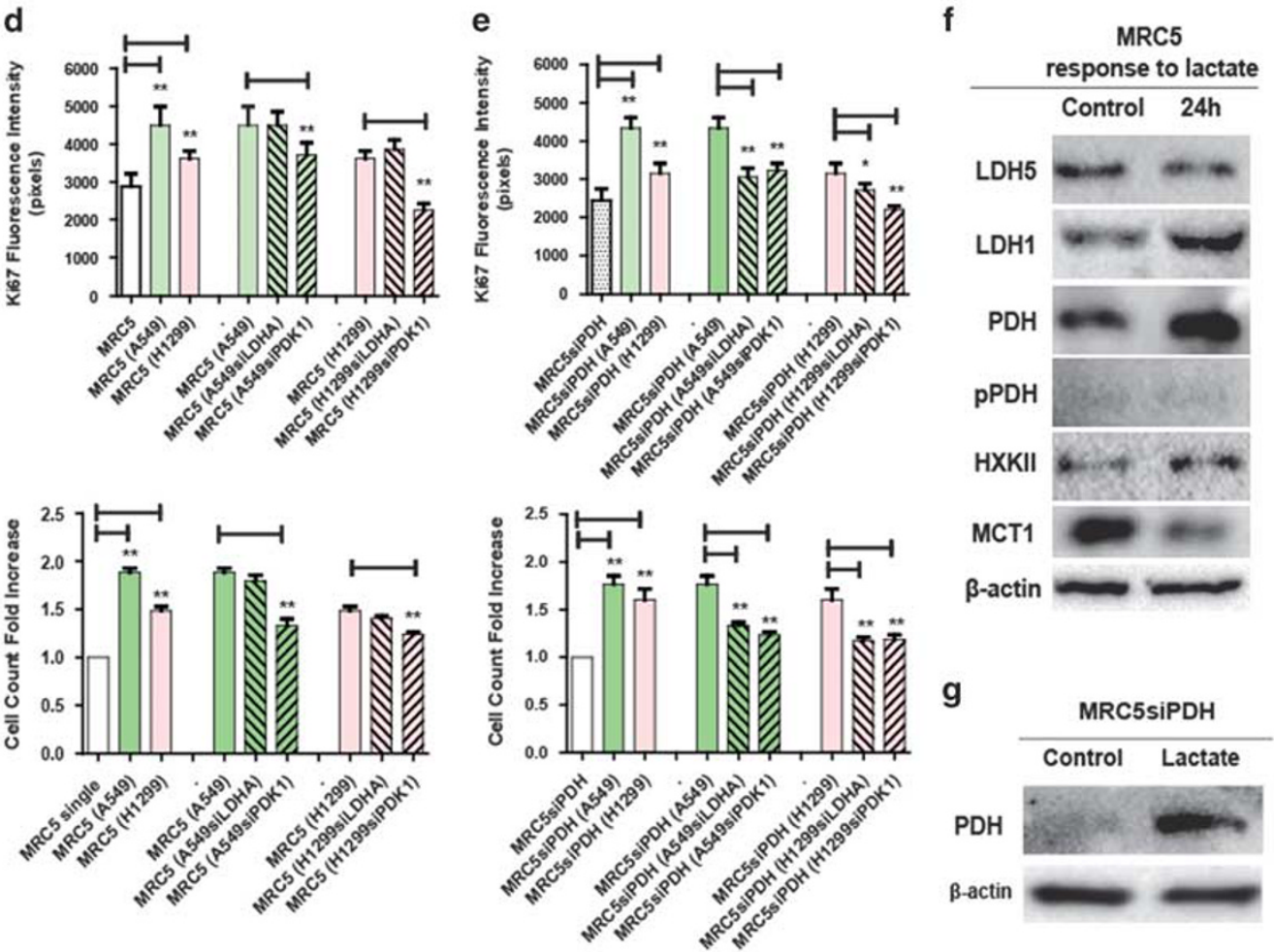

Figure 3 (a) Fold change of cell density (Trypan Blue assay) after 2-day culturing of MRC5 fibroblast and A549 or H1299 lung cancer cells in sibling cultures or dissimilar co-cultures (sharing the same culture medium). (b) Ki67 proliferation marker expression in the nuclei of MRC5 fibroblasts and A549 and $\mathrm{H} 1299$ cancer cells when growing in sibling cultures or dissimilar co-cultures (sharing the same culture medium) and densitometry analysis. (c) Western blotting images showing effective suppression of LDHA and PDK1 protein expression in A549 and H1299 lung cancer cell lines and of the PDH in MRC5 fibroblasts, using gene-specific pools of siRNAs. (d) Fluorescence intensity of nuclear Ki67 and cell density assessed by the Trypan Blue assay in sibling MRC5 cultures and in dissimilar co-cultures ( ${ }^{*} P$-value $<0.01$ refers to comparison as shown by the bars above the columns). (e) Fluorescence intensity of nuclear Ki67 and cell density assessed by the Trypan Blue assay in sibling cultures of MRC5 fibroblasts with silenced PDH gene vs in dissimilar co-cultures with A549 and H1299 lung cancer cells (wild type or with silenced LDHA or PDK1 genes) $\left({ }^{*} P\right.$-value $>0.05$, ${ }^{* *} P$-value $<0.01$ refer to comparison as shown by the bars above the columns). (f) Changing of metabolic profile of MRC5 fibroblasts following $24 \mathrm{~h}$ incubation with lactate, as assessed with western blotting. (g) Western blotting detection of PDH in MRC5 fibroblasts with silenced PDH cultured for $24 \mathrm{~h}$ after addition of lactate to the culture medium $(1 \mu \mathrm{l} / \mathrm{ml})$.

cancer cell lines exhibited a higher Ki67 nuclear presence when co-cultured in common medium, as shown in confocal microscopy (Figure 3b).

To investigate the effect of key metabolism enzymes on cell proliferation in co-cultures, the LDHA and PDK1 genes were silenced in cancer cells, while the PDH gene was silenced in MRC5 fibroblasts (Figure 3c). Suppression of cancer cell anaerobic potential by silencing of the LDHA gene followed by co-culturing with fibroblasts showed that the stimulatory effect on fibroblasts persisted (Figure 3d). Silencing of PDK1 gene in cancer cells, however, reduced their stimulatory effect on fibroblast proliferation (Figure 3d). This was confirmed in both Ki67 and cell count analysis.

Further experiments on fibroblast with suppressed $\mathrm{PDH}$ (suppression of aerobic potential) showed that these continued to experience increased proliferation ability when co-cultured with A549 and the H1299 cancer cell lines (Figure 3e). In contrast, suppression of the cancer cell 
anaerobic metabolism with siLDHA or siPDK resulted in the reduction of the stimulatory effect exerted by tumor cells on the proliferation of siPDH fibroblasts (Figure 3e).

\section{The Effect of Lactate on MRC5 Fibroblasts}

Twenty-four-hour incubation of MRC5 fibroblasts with lactate $(1 \mu \mathrm{l} / \mathrm{ml}$ from $85 \% \mathrm{w} / \mathrm{w}$ DL-Lactic acid; L1250; Sigma Aldrich) resulted in significant changes of the metabolic profile, showing an increase of LDHB and of PDH, suggestive of a response toward intensified lactate reverse transformation to pyruvate and increased pyruvate usage in the Krebs cycle (Figure 3f). This may also explain why partial silencing of the PDH gene in fibroblasts did not prevent the stimulatory effect from cancer cells, presumably due to $\mathrm{PDH}$ expression stimulation that overcomes the effect of silencing. Indeed, the expression of PDH in MRC5 cell with silenced PDH was clearly increased when cells were cultured in lactate acidic environment (Figure 3g) Phosphorylated PDH levels were very low, and hexokinase levels were stable, while monocarboxylate transporter MCT1 levels decreased, presumably as a protective pathway to reduce lethal intracellular acidosis.

\section{Cancer Cell Proliferation in Co-Cultures with Fibroblasts} The nuclear expression of Ki67 proliferation marker of tumor cells, as assessed with confocal microscopic image analysis, was increased when A549 or H1299 cells were cultured in common medium with fibroblasts. This effect was maintained even when the PDH gene was suppressed in fibroblasts (Figures $4 \mathrm{a}$ and $\mathrm{b}$ ). This finding, however, may have been a result of the previously observed PDH increase in co-cultured fibroblasts or cultured under lactate presence, which occurs even when PDH is partially silenced by siRNA techniques. Suppression of LDHA or PDK1 gene in either of the cancer cell lines did not affect their proliferation capacity, and cells experienced increased proliferation when co-cultured with fibroblasts. The silencing of the $\mathrm{PDH}$ gene, however, in fibroblasts prevented the stimulatory effect on tumor cells with silenced LDHA or PDK1 (Figures $4 \mathrm{a}$ and $\mathrm{b}$ ).

H1299 cells growing in the metastatic foci within the MRC5 fibroblast area showed an intense 100\% expression of the Ki67 proliferation marker (Figure 4c).

\section{Cancer Cell Migration Toward Fibroblast Areas}

To assess the effect of fibroblasts and of metabolism gene expression in the migratory ability of A549 and H1299 lung cancer cells, migration experiments were performed using the IBIDI Single Culture-Insert assay. In order to exclude proliferation of cells as a confusing factor, the migration assay was applied with and without incubation of cells with Mitomycin C. The filling of gap followed identical rates as compared with untreated cells (data not shown). The migration rates of A549 and H129 lung cancer cells seemed not to change when the filling of gap was assessed against the same type of cancer cells or against MRC5 fibroblasts (data not shown).
Silencing, however, of PDH gene in fibroblasts or silencing of PDK1 or LDHA gene in tumor cells or even couple silencing of these genes in fibroblasts and cancer cells led to a considerable delay of cancer cell migration toward the fibroblast area (Figures 4d-f). The A549 cancer cell migration was most potently inhibited when PDK1 was suppressed in parallel with the inhibition of $\mathrm{PDH}$ expression in fibroblasts (Figure 4f). In H1299 cell line, maximal migration inhibition was achieved by the simultaneous inhibition of the expression of PDH in fibroblasts and LDHA in tumor cells (Figure 4f).

\section{DISCUSSION}

In the current study, we investigated the metabolic adaptation of lung fibroblasts and of lung cancer cells when growing separately within the same culture medium, allowing therefore paracrine interactions. Indeed, significant and differential changes were confirmed, as early as $24 \mathrm{~h}$ after cell lines were brought in contact. GLUT1, responsible for glucose absorption, is intensified in the two cancer cell lines, reflecting increasing energy demands, presumably in the context of an intensified growth. Normal MRC5 fibroblasts upregulated the expression of the non-phosphorylated $\mathrm{PDH}$, suggesting an intensification of the aerobic consumption of glucose through pyruvate production and introduction into the Krebs cycle. In contrast, cancer cells adjusted the expression of key enzymes related to anaerobic glycolysis. Lactate dehydrogenase LDH5 (composed of LDHA subunits) was upregulated in cancer cells, while LDHB was suppressed, reflecting an intense transformation of glucose to pyruvate and, thereafter, to lactate. In parallel, the PDH kinase PDK1 was upregulated, showing a forced phosphorylation and deactivation of PDH, further blocking the transformation of pyruvate to acetylCoA, thus blocking the main fuel for mitochondrial respiration in cancer cells. Indeed, the levels of phosphorylated PPDH were strongly increased in cancer cells but not in normal fibroblasts. Finally, the monocarboxylate transporter MCT1 levels were upregulated in both cancer cells and normal fibroblasts. As these transporters work according to the concentration gradient of lactate, it is postulated that MCT1 favors extrusion of the overproduced lactate outside the cells into the medium to avoid intracellular acidosis and death. Fibroblast, on the other hand, sustained high expression levels of LDH1 (composed of LDHB-subunits), an enzyme that favors lactate transformation back to pyruvate. Thus MCT1 upregulation in fibroblasts may show intensified absorption of the lactate released by cancer cells, presumably to be used as a metabolic fuel, at the same time buffering the suppressive and lethal condition of lactic acidosis in the medium.

Studying the growth rates of normal fibroblasts and cancer cells in co-cultures, we noted an intensified growth of both compartments when cancer cells were cultured aside to lung fibroblasts for 2 days. This is in accordance with a study by Majeti et al ${ }^{14}$ reporting increased proliferation in $3 \mathrm{D}$ co-cultures of various cancer cells with fibroblasts. Moreover, 
a

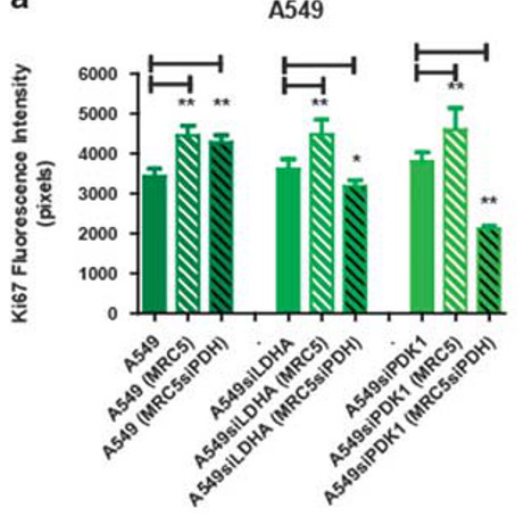

b

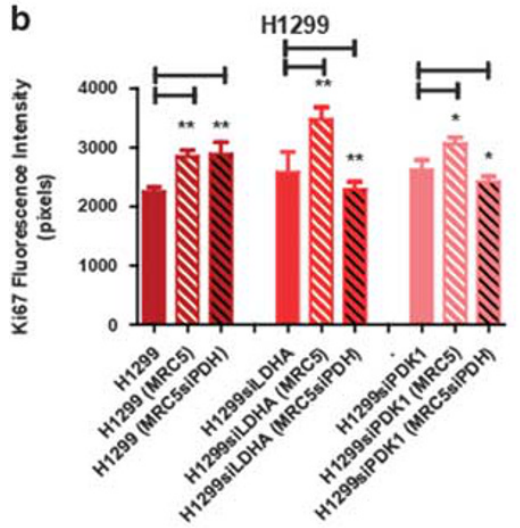

d

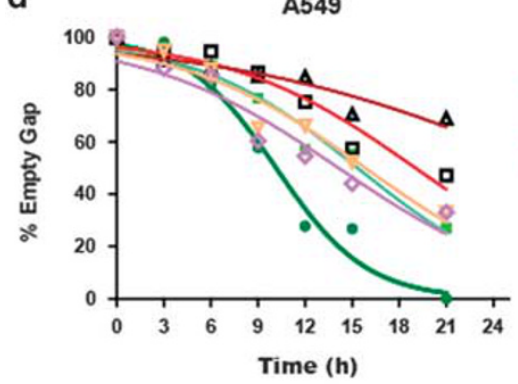

e

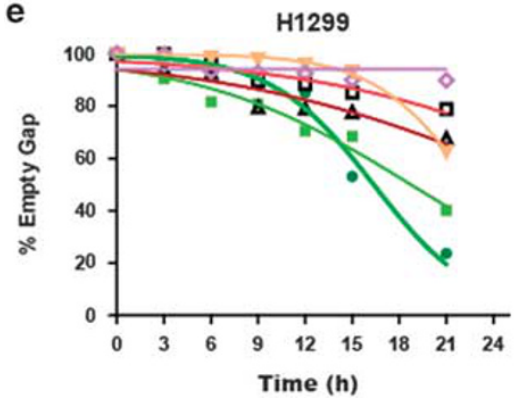

A549

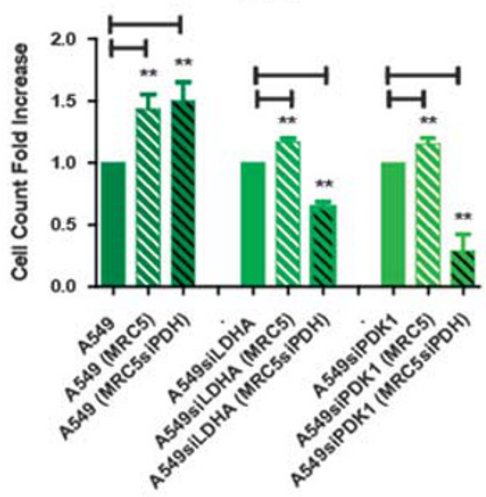

C $\quad \mathrm{KI} 67$ nuclear prollferation marker
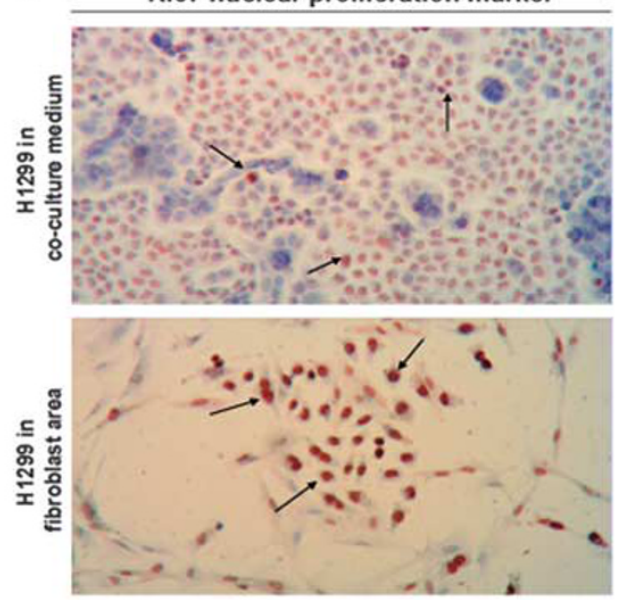

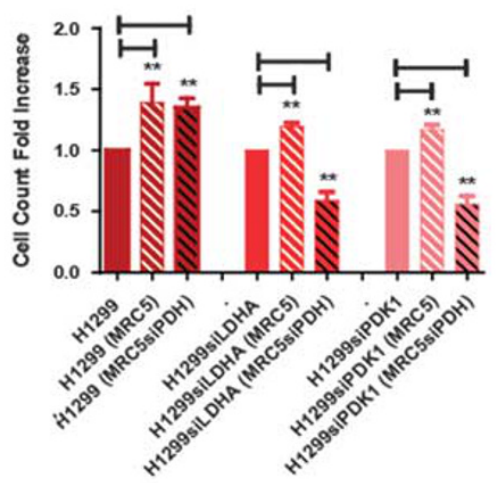

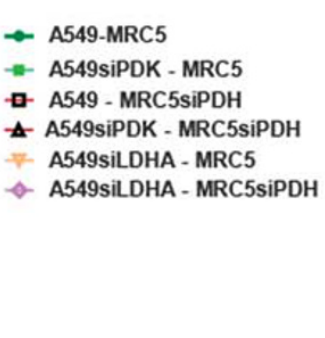

- H1299 - MRC5

- H1299siPDK - MRC5

a- H1299-MRC5siPDH

$\triangle$ H1299siPDK - MRC5siPDH

7- H1299siLDHA - MRC5

- H1299siLDHA - MRC5siPDH

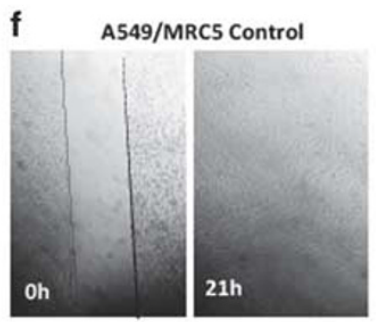

H1299/MRC5 Control

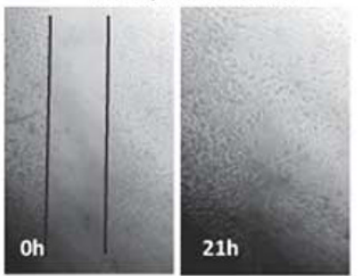

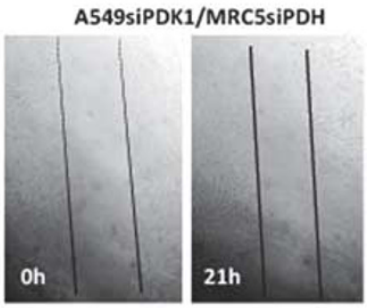

H1299siLDHA/MRCSsiPDH

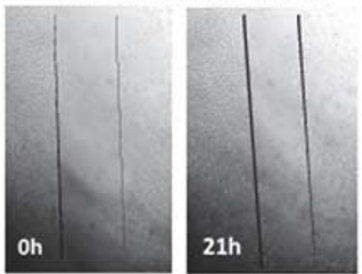

Figure $4(\mathbf{a}, \mathbf{b})$ Confocal image analysis of fluorescence intensity of nuclear Ki67 proliferation marker expression and of cell density assessed by the Trypan Blue assay in cancer cells A549 (a) and H1299 (b) with or without LDHA or PDK1 gene silencing cultured for 2 days in culture medium as sibling cultures or dissimilar co-cultures with fibroblasts MRC5 (with or without the PDH gene silencing). Asterisks show the $P$-values $\left({ }^{*}<0.05,{ }^{* *}<0.01\right.$ ) referring to comparisons as shown by the bars above the columns. (c) Immunocytochemical image of the 'metastasis-like' H1299 cell nest growing in the MRC5 fibroblast area, showing extensive and intense nuclear expression. (d-f) Inhibition of cell migration of cancer cells after silencing of genes in cancer cells (LDHA or PDK1) and/or fibroblasts (PDH). Graphical presentation of the percentage of gap filling in time (hours) is shown in panel (d) for A549 and panel (e) for H1299. (f) Microscopic images of the gap from selected cell combinations before removal of silicon insert and $21 \mathrm{~h}$ later. 
mesenchymal stromal cells have been shown to stimulate breast cancer cell growth, a result that, at least in part, is related to soluble factors excreted by the mesenchymal cells. ${ }^{15}$ Partial silencing of PDH in fibroblasts did not affect the stimulatory effect of cancer cells on fibroblast cell proliferation. This, however, was shown to be a result of abrogation of the silencing effect by the lactate produced by cancer cells (lactate induces $\mathrm{PDH}$ in fibroblasts treated with siPDH). Suppression of the cancer cell anaerobic metabolism resulted in the reduction of the stimulatory effect exerted by tumor cells on the proliferation of fibroblasts, but this was statistically confirmed only for siPDK1. The silencing of PDK1 or of LDHA in cancer cells resulted in significantly decreased proliferation of MRC5 fibroblasts that had silenced $\mathrm{PDH}$ expression. Similarly, the silencing of the PDH gene in fibroblasts attenuated the stimulatory effect on tumor cells only when the LDHA or PDK1 gene had been suppressed. This shows that a combination of active aerobic fibroblast metabolism and anaerobic cancer cell metabolism is necessary for the stimulatory interaction between cell types on cell proliferation to appear. These findings support an important role of the anaerobic glycolytic activity in cancer cells and of the aerobic activity in fibroblasts to mutually stimulate their growth.

Whether lactate and related metabolism byproducts are involved in this interactive signaling is unknown. In a study by Wagner et al, ${ }^{16}$ exogenous lactate induced proliferation of cultured fibroblast through oxidant production. We failed, however, to show a direct stimulatory effect of exogenous lactate on MRC5 naive fibroblasts (data not shown), which may suggest that lactate-related metabolism byproducts or other anaerobic metabolism-released molecules may be involved. Nevertheless, $24 \mathrm{~h}$ incubation of MRC5 fibroblasts with lactate resulted in significant changes of the metabolic profile, showing an increase of LDHB and of $\mathrm{PDH}$, suggestive of a response toward intensified lactate reverse transformation to pyruvate and increased pyruvate usage in the Krebs cycle.

Studies in the literature support that CAFs induce an increased motility of lung cancer cells in co-culture assays. ${ }^{17}$ Tumor-associated fibroblasts also enhance invasive migration in breast cancer and enable transmigration through endothelial brain cells. ${ }^{18}$ In a recent study, Shiraishi et al ${ }^{19}$ showed that high aerobic glycolytic rates are important for cancer cell cytoskeletal remodeling and faster migration. This could not be compensated by the cancer cell oxidative phosphorylation activity when aerobic glycolysis was suppressed. Moreover, the acidity provided by lactate production seems to be important for local invasive activity of cancer cells. ${ }^{20}$ In the current study, we showed that silencing of $\mathrm{PDH}$ gene in fibroblasts or silencing of PDK1 or LDHA gene in tumor cells or even couple silencing of these genes in fibroblasts and cancer cells led to a considerable delay of cancer cell migration. It is, therefore, suggested that an intact aerobic metabolism of tumor-associated fibroblasts support the glycolysis-stimulated cancer cell's migratory activity.
Taking the above into account, we conclude that metabolic cooperation is rapidly triggered through paracrine pathways between lung cancer cells and lung fibroblasts. A direct Warburg effect is supported, as fibroblasts, through this interaction, are driven to intensified aerobic usage of glucose and/or lactate, while cancer cells reinforce their anaerobic glycolytic activity, despite the presence of oxygen (aerobic glycolysis). This metabolic cooperation between lung cancer cells and fibroblasts promotes proliferation of both cell compartments and cancer cell migration. For this phenomenon to occur, anaerobic metabolism of cancer cells, as shown by the critical role of the LDHA and PDK1 genes, and a functional Krebs' cycle in fibroblasts with intact $\mathrm{PDH}$ activity is demanded. Whether lactate or lactate-related activity or even other byproducts of anaerobic glucose metabolism are involved in the signaling mechanism of such intercommunication demands further investigation.

This in vitro study is in accordance with previous pathological studies of ours, where most tumors showed increased expression of anaerobic metabolism enzymes in cancer cells in a background of an aerobic adjacent stroma. ${ }^{10,11}$ However, as tumors are complex biological systems, the prevalence of the reverse Warburg effect in at least a subset of human carcinomas cannot be excluded.

Supplementary Information accompanies the paper on the Laboratory Investigation website (http://www.laboratoryinvestigation.org)

\section{ACKNOWLEDGMENTS}

The study was funded by the Lifelong Learning - Aristeia Project, project, code no. 4046, ESPA 2007-2013, GGET decision number 1532/10-2-14. Additional funding was obtained from the 81006 project of the Special Account of Democritus University of Thrace.

\section{DISCLOSURE/CONFLICT OF INTEREST}

The authors declare no conflict of interest.

1. Macheda ML, Rogers S, Best JD. Molecular and cellular regulation of glucose transporter (GLUT) proteins in cancer. J Cell Physiol 2005;202: 654-662.

2. Warburg O. On the origin of cancer cells. Science 1956;123:309-314.

3. Gatenby RA, Gillies RJ. Why do cancers have high aerobic glycolysis? Nat Rev Cancer 2004;4:891-899.

4. Epstein T, Xu L, Gillies RJ, et al. Separation of metabolic supply and demand: aerobic glycolysis as a normal physiological response to fluctuating energetic demands in the membrane. Cancer Metab 2014;2:7.

5. Lunt SY, Vander Heiden MG. Aerobic glycolysis: meeting the metabolic requirements of cell proliferation. Annu Rev Cell Dev Biol 2011;27: 441-464.

6. Halestrap AP, Price NT. The proton-linked monocarboxylate transporter (MCT) family: structure, function and regulation. Biochem J 1999;2: 281-299.

7. Koukourakis MI, Giatromanolaki A, Sivridis E, et al. LYVE-1 immunohistochemical assessment of lymphangiogenesis in endometrial and lung cancer. J Clin Pathol 2005;58:202-206.

8. Sivridis E, Giatromanolaki A, Koukourakis MI. "Stromatogenesis" and tumor progression. Int J Surg Pathol 2004;12:1-9.

9. Giatromanolaki A, Koukourakis MI, Koutsopoulos A, et al. The metabolic interactions between tumor cells and tumor-associated stroma (TAS) in prostatic cancer. Cancer Biol Ther 2012;13:1284-1289. 
10. Koukourakis Ml, Giatromanolaki A, Bougioukas $\mathrm{G}$, et al. Lung cancer: a comparative study of metabolism related protein expression in cancer cells and tumor associated stroma. Cancer Biol Ther 2007;6: 1476-1479.

11. Koukourakis Ml, Giatromanolaki A, Harris $\mathrm{AL}$, et al. Comparison of metabolic pathways between cancer cells and stromal cells in colorectal carcinomas: a metabolic survival role for tumorassociated stroma. Cancer Res 2006;66:632-637.

12. Martinez-Outschoorn UE, Pavlides S, Howell A, et al. Stromal-epithelial metabolic coupling in cancer: integrating autophagy and metabolism in the tumor microenvironment. Int J Biochem Cell Biol 2011;43: 1045-1051.

13. Kim SA, Lee EK, Kuh HJ. Co-culture of 3D tumor spheroids with fibroblasts as a model for epithelial-mesenchymal transition in vitro. Exp Cell Res 2015;335:187-196.

14. Majety $M$, Pradel $L P$, Gies $M$, et al. Fibroblasts influence survival and therapeutic response in a 3D co-culture model. PLoS ONE 2015;10: e0127948.
15. Pasanen I, Pietilä M, Lehtonen $S$, et al. Mesenchymal stromal cells from female donors enhance breast cancer cell proliferation in vitro. Oncology 2015;88:214-225.

16. Wagner $\mathrm{S}$, Hussain $\mathrm{MZ}$, Hunt $\mathrm{TK}$, et al. Stimulation of fibroblast proliferation by lactate-mediated oxidants. Wound Repair Regen 2004;12:368-373.

17. Kim SH, Choe C, Shin YS, et al. Human lung cancer-associated fibroblasts enhance motility of non-small cell lung cancer cells in co-culture. Anticancer Res 2013;33:2001-2009.

18. Choi YP, Lee JH, Gao MQ, et al. Cancer-associated fibroblast promote transmigration through endothelial brain cells in three-dimensional in vitro models. Int J Cancer 2014;135:2024-2033.

19. Shiraishi T, Verdone JE, Huang J, et al. Glycolysis is the primary bioenergetic pathway for cell motility and cytoskeletal remodeling in human prostate and breast cancer cells. Oncotarget 2015;6:130-143.

20. Estrella V, Chen T, Lloyd $M$, et al. Acidity generated by the tumor microenvironment drives local invasion. Cancer Res 2013;73: 1524-1535. 